\title{
Non-locality and the misdiagnosis of Spinal Cord Injury Without Radiographic Abnormality: proof of concept
}

\author{
Kevin Rolfe ${ }^{1,2} \cdot$ Aaron Beck $^{2} \cdot$ Tracy Kovach $^{2} \cdot$ Brian Mayeda $^{3} \cdot$ Charles Liu Li, $^{1,4}$
}

Received: 27 October 2018 / Revised: 18 December 2018 / Accepted: 28 December 2018

(c) International Spinal Cord Society 2019

\begin{abstract}
Study Design This is a retrospective review.

Objectives To validate the concept of "non-locality" to explain cases of Spinal Cord Injury Without Radiographic Abnormality (SCIWORA) previously deemed inexplicable. To investigate and challenge the source data for the SCIWORA hypothesis which has the built-in assumption that a traumatic spinal cord injury (SCI) can only be caused by a local or adjacent spinal column injury and which, therefore, postulates that the pediatric spinal column is inherently more flexible than the spinal cord to explain SCI whenever a local spinal column injury is not detected.

Setting A National Rehabilitation Center, one of fourteen which reports to the Spinal Cord Injury Model System.

Methods We examined all residual SCIWORA cases over a 5-year period. In addition, we performed an extensive literature search to trace the evidence supporting the SCIWORA hypothesis that children's spinal columns are inherently lax and may stretch more than the spinal cord prior to disruption.

Results Six SCI patients with a residual diagnosis of SCIWORA were identified, 3 pediatric and 3 adult. All had injuries fitting non-locality. None were an actual SCIWORA. Source data do not appear to support the SCIWORA hypothesis.

Conclusion Borrowing from quantum mechanics, we reveal non-locality as a real entity in the spine. The assumption of locality-only is invalid and likely contributed to the SCIWORA hypothesis for the pediatric spine. Misdiagnosis and misunderstanding of SCIWORA may lead to improper treatment and increased cost. Awareness may facilitate search for adequate explanations for difficult cases rather than mere assignment as SCIWORA.
\end{abstract}

\section{Introduction}

Spinal Cord Injury Without Radiographic Abnormality (SCIWORA) was coined by Pang in 1982 for children who sustained a traumatic spinal cord injury (SCI) without radiographic evidence of an adjacent spinal column injury to explain the SCI [1]. SCIWORA has the built-in assumption

Kevin Rolfe, MD, MPH

kwrolfe@gmail.com

1 Rancho Los Amigos National Rehabilitation Center, 7601 E. Imperial Highway, Downey, CA 90242, USA

2 Harbor-UCLA Medical Center, 1000W. Carson St, Torrance, CA 90509, USA

3 Cedars-Sinai Medical Center, 8700 Beverly Blvd, Los Angeles, CA 90048, USA

4 University of Southern California, 1500 San Pablo St, Los Angeles, CA 90033, USA "where the SCI occurs, there the spinal column injury occurs" (or thereabouts). Since SCIWORA pre-dates widespread magnetic resonance imaging (MRI) use, many believed MRI would provide a missing nearby soft-tissue explanation, and it did, but not for all, and SCIWORA persisted. Though some recommend terms like SCIWONA (spinal cord injury without neuroradiologic abnormality) for modern cases without explanation despite all studies including MRI, the term has not caught on and SCIWORA continues to be applied loosely to all such cases $[2,3]$.

This paper challenges the assumption in traumatic SCI, which led to the SCIWORA hypothesis, i.e., the notion we call "locality" that states if any SCI occurs, then any causal spinal column injury must be in the immediate vicinity. Thus, to explain cases of SCI when no local spinal column injury is present, the SCIWORA hypothesis postulates that the pediatric spinal column is inherently lax, i.e., the spinal column has more physiologic flexibility than the spinal cord. We present a case series misdiagnosed as SCIWORA to demonstrate the existence of non-locality, meaning the 


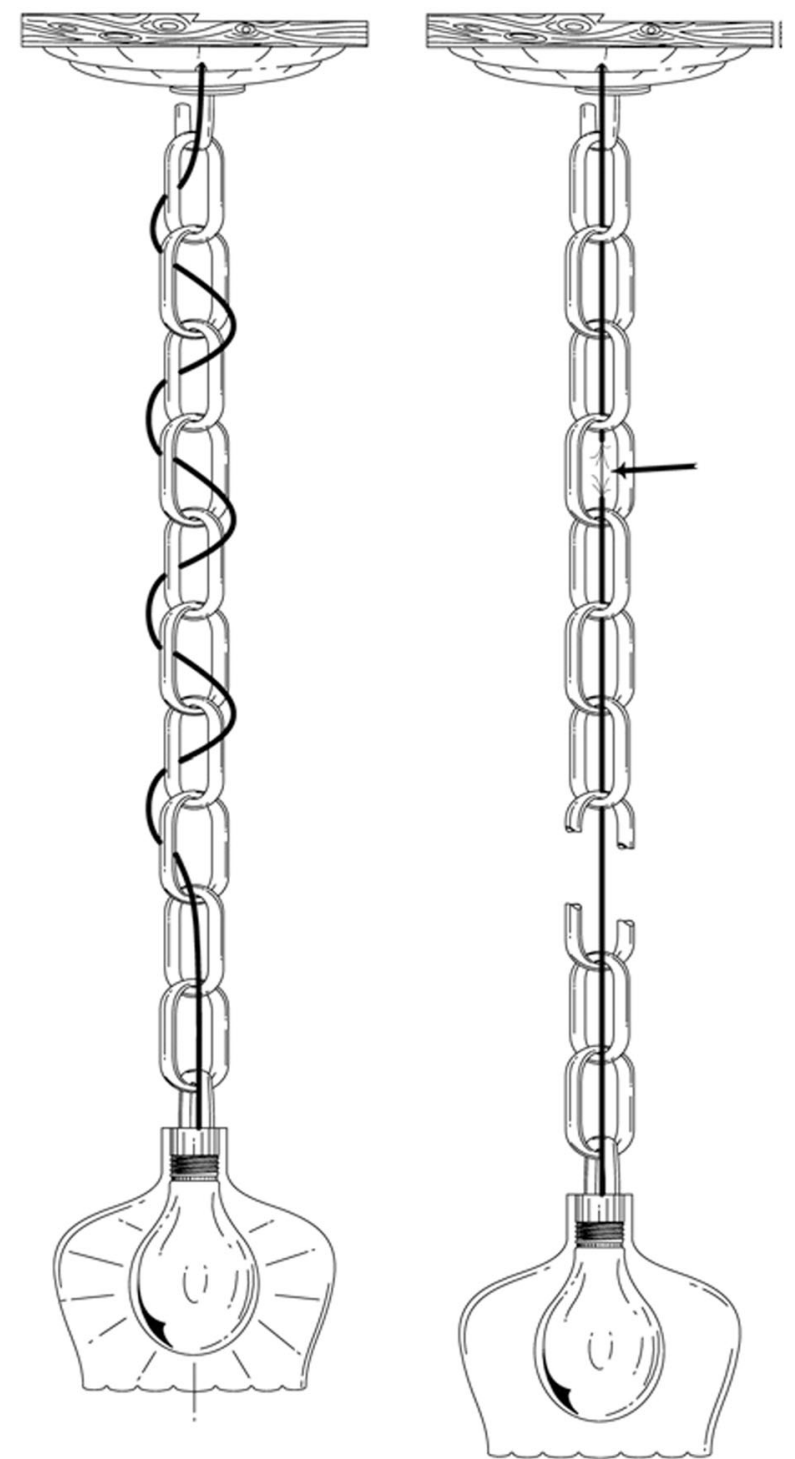

Fig. 1 An analogy for the non-locality effect. Imagine a light fixture suspended from the ceiling by a chain (spinal column) with its electrical cord (spinal cord) intertwined. If a single link of chain breaks, the supporting column is uncoupled and the entire length of electrical cord will come under distractive tension. Should the cord rupture (arrow), there is no reason for it to occur at the same level as the chain disruption; it would most likely occur at its own weakest link. Repair of the single link of chain would reconfer stability and protection of the cord. Extra support or bracing to the uninjured chain links would have no additional effects. (Courtesy of James Prinzivalli, reproduced with permission JBJS.) [6]

SCI and the spinal column uncoupling or damage need not occur in the same location (Fig. 1). So long as even one of these cases is true, then the real existence of non-locality is demonstrated, even if the majority of spinal column and spinal cord injuries do occur adjacent to one another. Last, the medical literature that has been cited as evidence for the SCIWORA hypothesis is examined for its actual supporting content.

\section{Methods}

Rancho Los Amigos is a National Rehabilitation Center for SCIs, pediatric and adult, and the spine team reviews and consults with each admission. SCI consults for the last 5 years (2012-2017) were screened for a diagnosis of SCIWORA. All SCIWORA cases underwent chart and radiographic review to determine accuracy or consistency with the diagnosis and whether the assumption of locality (i.e. that the spinal column injury, when identifiable, must occur near the level of the spinal cord injury) was confirmed. Neurological levels and severity of impairment were graded according to the American Spinal Injury Association International Standards for Neurological Classification of Spinal Cord Injury ASIA/ISCoS [4]. All patients sustained an American Spinal Injury Association Impairment Scale (AIS) grade of A and are referred to as "complete" hereafter. Institutional Review Board approval was obtained.

\section{Results}

Six SCIWORA patients were identified over a 5-year period: 3 pediatric (boy age 4, girl age 2, girl age 13) and 3 adult (female age 23 and males age 24 and 65). Five were restrained passengers in motor vehicle collisions (MVC) and 1 was a motorcycle accident (MCA) victim. All six involved sudden deceleration with a distractive mechanism implicated for the spinal column injury, but without significant local spinal column translation or local cord compression.

\section{Case 1}

A 4-year-old male forward-facing rear passenger restrained in a child's seat was injured when the vehicle hit a tree at freeway speeds. A C1-2 distraction injury caused a T2 complete paraplegia and was mislabeled a T2 SCIWORA as the treating physicians saw no evidence of spinal column injury near $\mathrm{T} 2$. The patient was sent to our facility with an unstable C1-2 ligamentous injury above his T2 SCI level. The case has been previously reported and some of the images reproduced herein (Figs. 2 and 3) [5]. The patient underwent successful $\mathrm{C} 1-2$ posterior spinal fusion to prevent subsequent risk of a secondary translation event at C1-2 which might render a C2 level SCI.

\section{CASE 2}

A 2-year-old girl in a forward-facing child's seat in a sudden deceleration MVC sustained a C6-7 distractive flexion injury with $\mathrm{C} 2$ complete $\mathrm{SCI}$ without nearby $\mathrm{C} 2$ level spinal column disruption locally on CT or MRI. In addition, there 

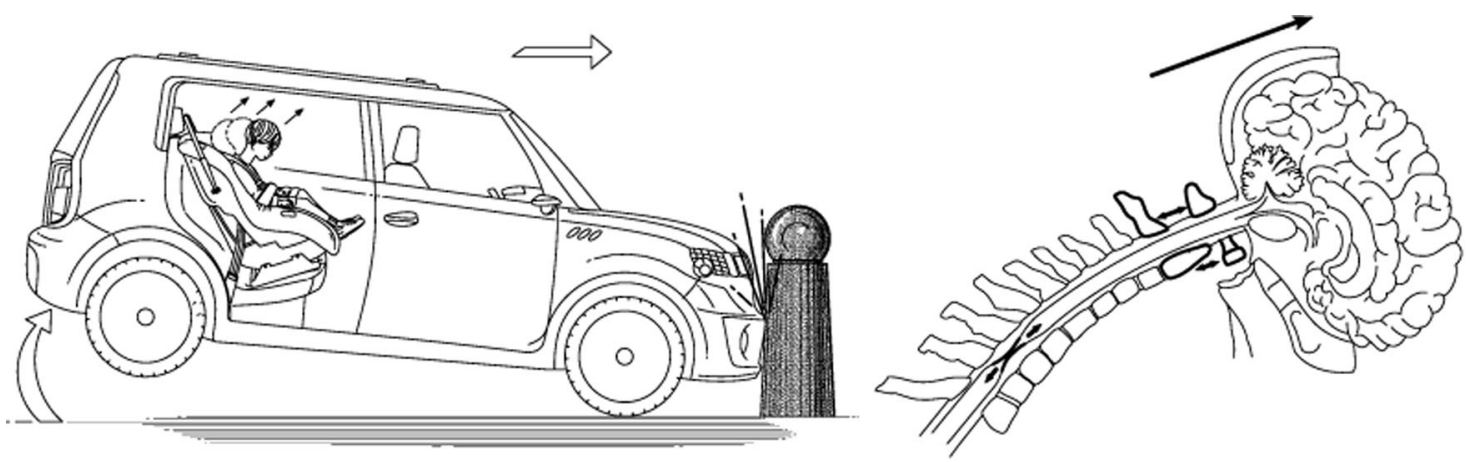

Fig. 2 Illustration depicting flexion-distraction injury of the spine. The spinal column was unhinged at the $\mathrm{C} 1-\mathrm{C} 2$ level, allowing the remaining inertial energy of the head to be dissipated by the spinal cord via distraction (without translation) at a caudal noncontiguous level. The mechanism is akin to pulling taffy from two ends, with the brain suspended by the skull base of the head at one end being carried away from the car-seat shoulder anchor at the other end. The intervening neurologic tissue may stretch anywhere along its length. (Reproduced with permission from JBJS and artist James Prinzivalli) [5]
A

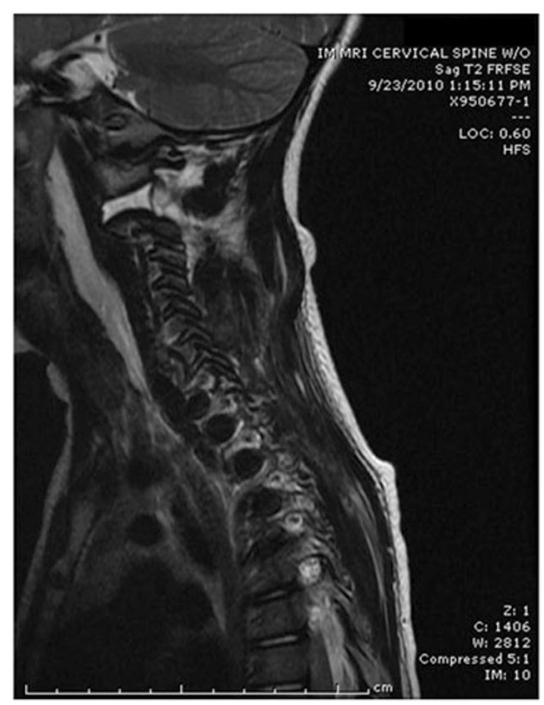

B

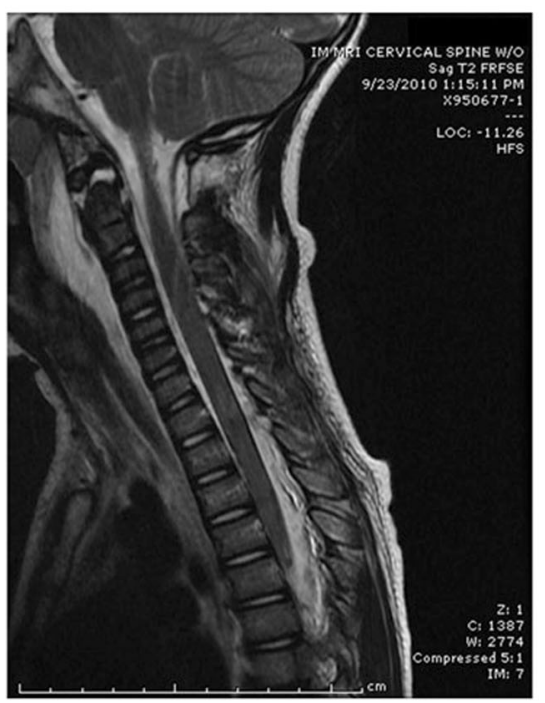

C

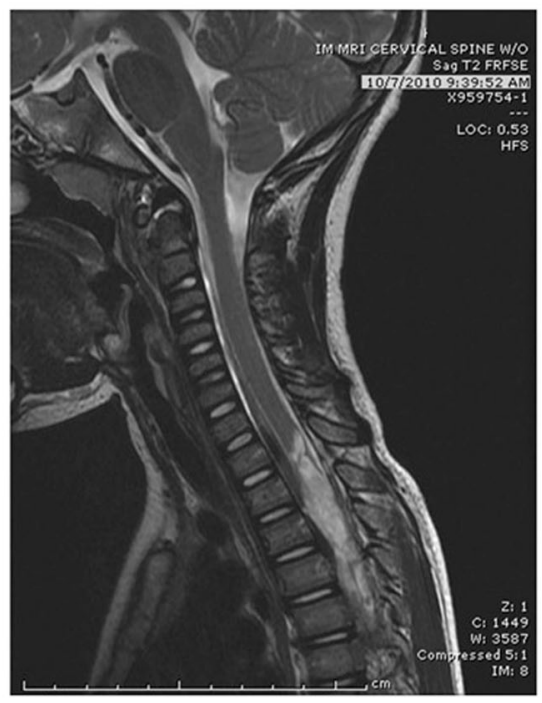

Fig. 3 Parasagittal T2-weighted MRI demonstrating residual $\mathrm{C} 1-\mathrm{C} 2$ joint distraction. The position of the involved structures reveals only the minimal amount of residual displacement after recoil, while the maximum displacement causing cord injury likely occurred only transiently during the accident (a). Initial post-injury mid-sagittal T2- weighted MRI showing little or no cord change despite T2 clinically complete paraplegia (b). Two-week follow-up midsagittal T2weighted MRI demonstrating abundant cord signal change at T2-T3 (c). (Reproduced with permission from JBJS) [5] was no C6-7 spinal cord injury adjacent to the C6-7 spinal column injury on CT or MRI (Fig. 4).

\section{CASE 3}

A twelve-year-old restrained rear passenger with shoulder harness was involved in a high-speed sudden deceleration MVC. She sustained a distractive-flexion C2 hangman's fracture (traumatic spondylolisthesis) and T2 clinical and radiologic SCI without evidence of T2 spinal column injury (Fig. 5).

\section{CASE 4}

A 65-year-old man in a sudden deceleration MVC sustained a T11-12 distraction injury with a C8 SCI without evidence of spinal column disruption about the level of the SCI and has been previously reported (Fig. 6) [6].

\section{Case 5}

A 24-year-old male MCA victim sustained a violent L2-3 distraction injury with clinical T9 SCI without nearby T9 spinal column disruption (Fig. 7). 

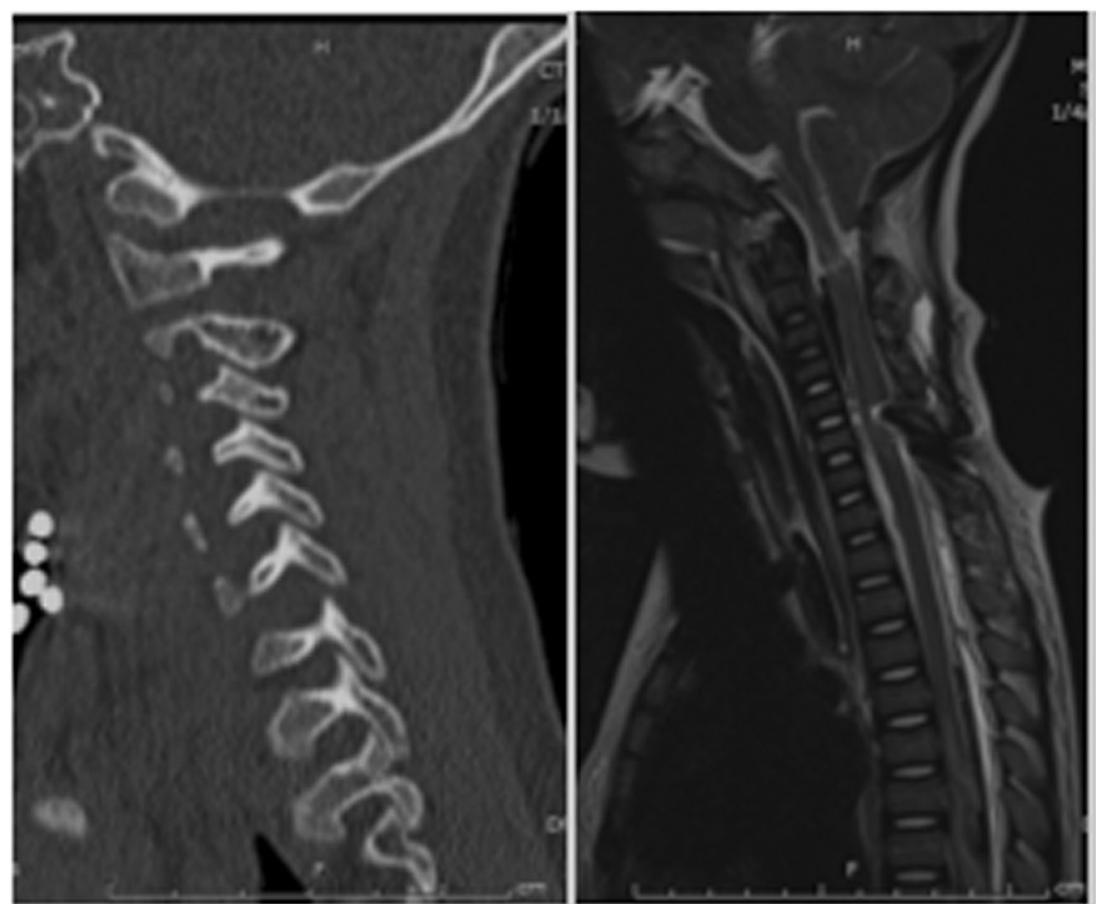

Fig. 4 Left: C6-7 flexion-distraction injury with C2 spinal cord injury. No disruption of the spinal column was seen on CT aside from the C6-7 injury. Note residual distraction and gapping of the C6-7 facet complex. The recoiled position of the involved structures reveals the minimal amount of residual displacement in the segment, while the maximum displacement causing cord injury likely occurred only

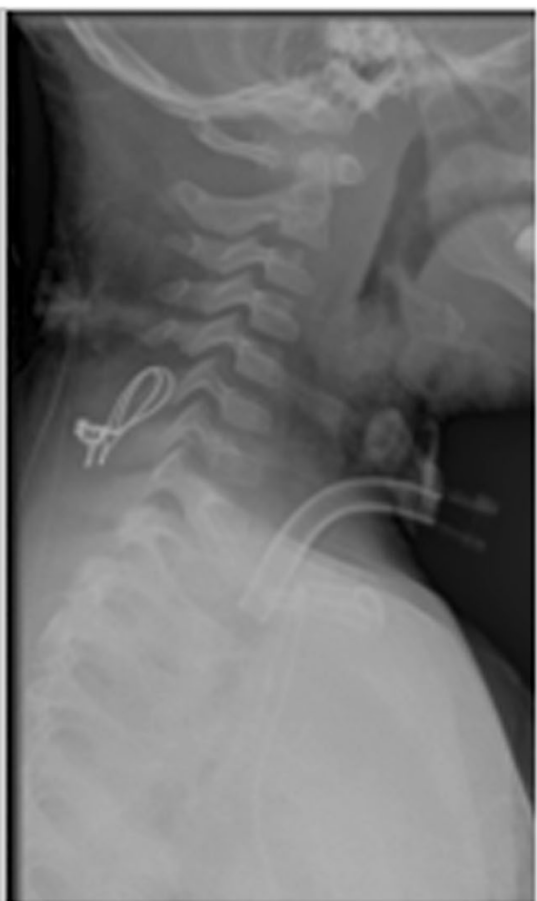

transiently during the accident. Middle: T2 sagittal MRI showing the C2 level spinal cord damage without local or adjacent osteoligamentous injury to explain the SCI. Tectorial membrane, dens, C1-2 vertebrae and articulation and occipitocervical joints were without injury. Right: Note post-operative MRI artifact at C6-7 after posterior spinous process wiring seen on $\mathrm{X}$-ray
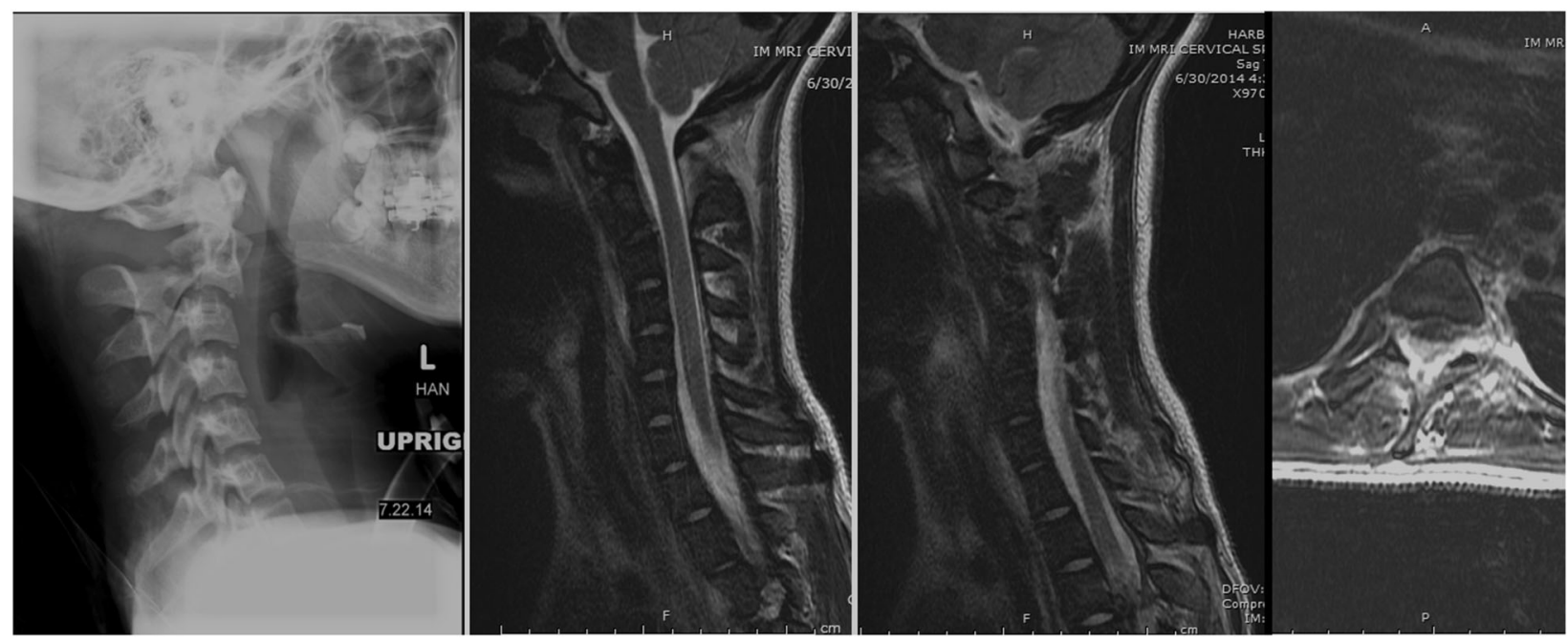

Fig. 5 Lateral radiograph (left) showing the residual displacement and C2 hangman's fracture after flexion-distraction motor vehicle collision. Sagittal MRI (middle left) showing normal spinal cord at C2 despite hangman's fracture. Sagittal and axial MRI (middle right and right) showing spinal cord injury at $\mathrm{T} 2$. No spinal column damage at $\mathrm{T} 2$ or any other region apart from the C2 hangman's injury was seen on CT or MRI despite complete imaging of brain, cervical, thoracic, and lumbo-sacral regions with each modality

\section{Case 6}

A 23-year-old female restrained passenger involved in a high-speed MVC sustained a distractive-flexion C2 hangman's fracture with a T1 cervicothoracic spinal cord injury without evidence spinal column osteoligamentous damage near the cervicothoracic junction and nearly identical to Case 3 (Fig. 8) 

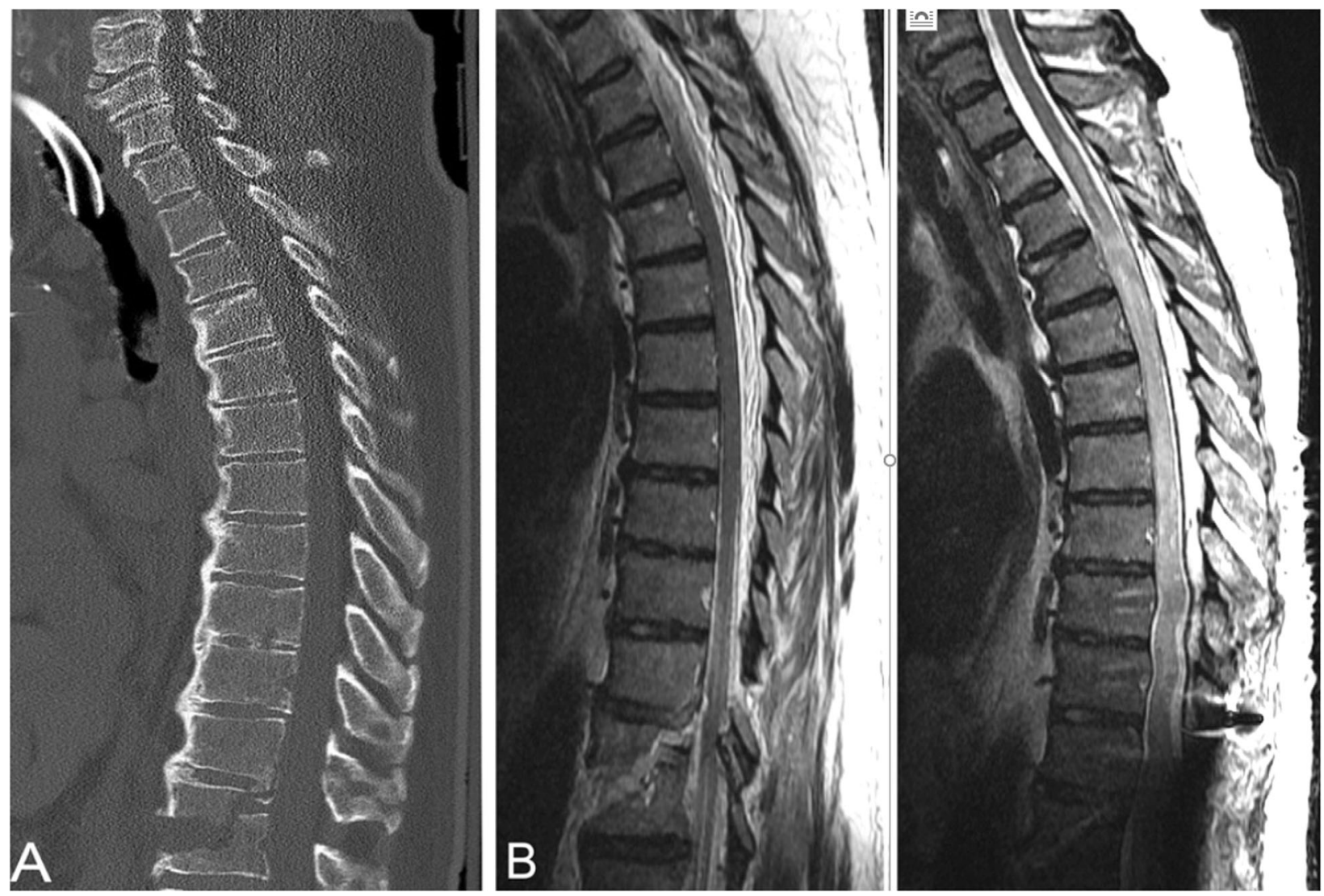

Fig. 6 a Initial sagittal CT showing the large residual displacement (not the actual maximal displacement that occurred transiently during the accident). Note the many thoracic-acquired autofusions. These immobile segments may have contributed in part to the pattern of diffuse cord stretch observed since they could not participate in distractive energy dissipation. b Initial T2-weighted sagittal MRI showing

\section{Discussion}

Borrowing from a similar concept in quantum mechanics, this series illustrates an effect we call "non-locality, "meaning a traumatic SCI may occur away from its causal spinal column injury, using a light fixture analogy and 6 representative cases (Fig. 1). If unconvinced by these cases, another example of the non-locality phenomenon has been present in the literature involving flexion-distraction Salter-Harris I odontoid fractures in children [7]. In 55 cases, 15 had SCI: 8 (53\%) occurred non-locally at the cervicothoracic junction, and 7 (47\%) occurred locally about C2. Non-local cervicothoracic SCI was more common than local C2 SCI. Though most SCIs, in general, result from a local spinal column injury, it appears that SCI may also result from a distant osteoligamentous uncoupling of the spinal column. With a positive case for non-locality in place, real explanatory power for previously mislabeled SCIWORA cases like these is available.

SCIWORA appears to have been born not simply from our early inability to visualize spinal column damage the T11-T12 injury. Note that there was little cord signal change despite clinically evident C8 tetraplegia. c Post-operative MRI at 6 weeks revealing the long cord stretch injury pattern beginning at the spinal column disruption level, extending cephalad, and tapering off at about T1. (Reproduced with permission from JBJS) [6].

radiographically, but also from our failure to recognize nonlocality. Pang has stated that any osteoligamentous MRI change seen for a SCIWORA "corresponds exactly to the level of the neurological lesion in each case" (p. 1333) [8]. From the assumption of locality-only effects, SCIWORA and its theories about the pediatric spinal column being more lax than the spinal cord were postulated in order to explain the missing spinal column damage that should have otherwise been expected. Even at face value, however, children have far fewer spinal cord injuries than adults despite much more vigorous physical activity, daily falls and so forth which presents common sense empirical evidence against such theories.

Given this, we set out to find the actual empirical evidence in the scientific literature that was used to justify the claims of SCIWORA. Two basic claims are made repeatedly:

1. The general claim that children's spines are inherently lax such that the spinal column can stretch more than the spinal cord prior to disruption;

-and- 


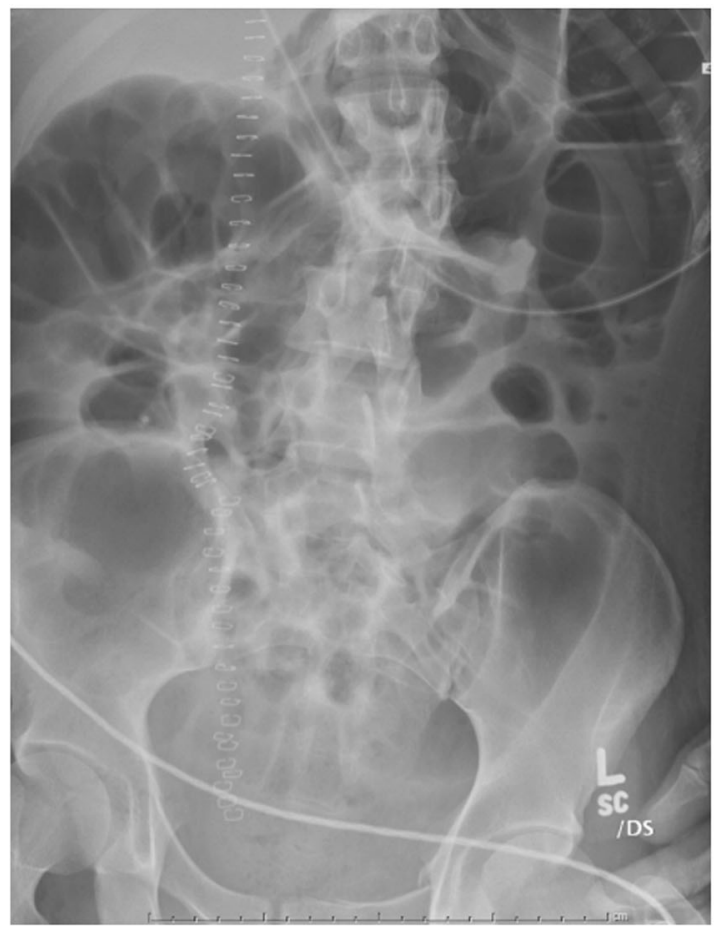

Fig. 7 Anteroposterior radiograph (left) showing violent residual displacement of spinal column at L2-3 after motorcycle accident. Sagittal CT (middle) showing flexion-distraction Chance fracture variant.

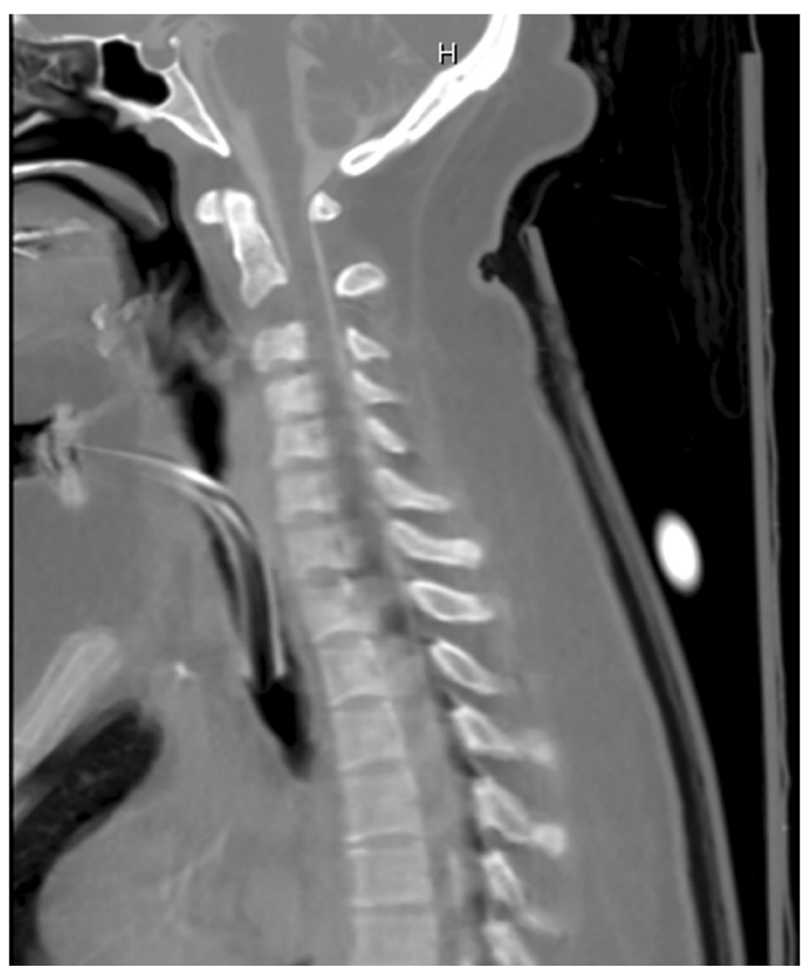

Fig. 8 Midsagittal CT showing distractive-flexion variant of $\mathrm{C} 2$ Hangman's fracture (traumatic spondylolisthesis) with clinical C8 complete SCI. No osteoligamentous injuries found on or about cervicothoracic junction. Note tipping forward of $\mathrm{C} 2$ representing residual minimal displacement
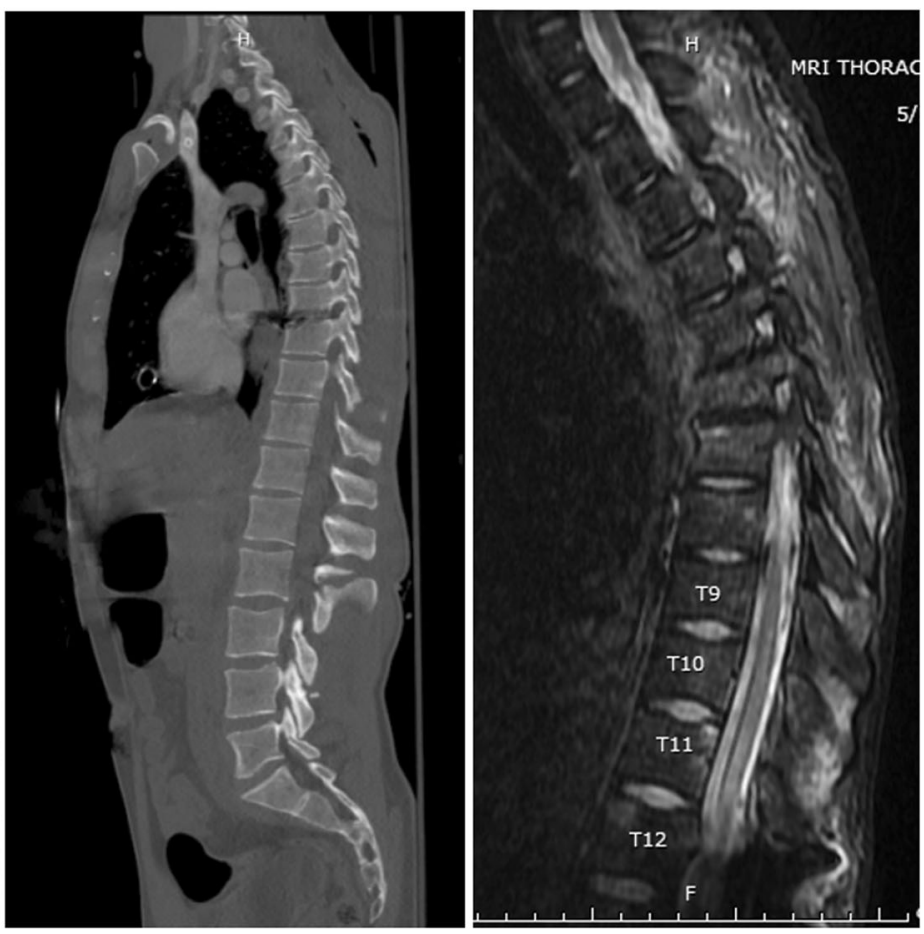

Sagittal MRI (right) showing tapering cord signal change up to T9 which was not evident on initial MRI

2. The specific claim that the spinal column can stretch 2 inches $(5.08 \mathrm{~cm})$ while the spinal cord can stretch only $1 / 4$ inch $(0.64 \mathrm{~cm})$ prior to disruption.

References of all PubMed articles with Spinal Cord Injury Without Radiographic Abnormality in the title for the last 10 years plus any written by Pang were traced back to their origin looking for any study along the path with the actual empirical data to support either of the claims.

For the specific claim that the spinal column may stretch physiologically up to 2 inches $(5.08 \mathrm{~cm})$ without disruption while the spinal cord may only stretch up to $1 / 4$ inch $(0.64 \mathrm{~cm}$, eight times less) before disruption, all reference trails lead to a single case series about 6 SCI breech births by Leventhal in 1960 [9]. One baby died and underwent autopsy. A single sentence in the discussion then appears in the entire 4-page paper making the claim: "It was shown in the autopsy specimen that this column can be stretched 2 inches and the cervical cord pulled down 1/4 inch" without any description of the methods employed.

For the general claim, 11 terminal references emerged, which were grouped into the following 4 categories excluding dead ends: (1) parallel breech births [10, 11], (2) normal pediatric spinal and radiographic anatomy and variants [12-15], (3) adults with cervical stenosis sustaining a SCI due to ligamentum flavum buckling after a hyperextension injury, but some without spinal column injury $[16,17]$, and (4) case series without static x-ray evidence to 
Table 1 Terminal articles cited for the SCIWORA hypothesis (excludes dead-ends and case series assuming the hypothesis a priori)

Leventhal 1960

Abroms et al. 1973

Bresnan et al. 1973

Bailey 1952

Cattell et al. 1965

Townsend et al. 1952

Sullivan et al. 1958

Taylor 1951

Marar 1974

Burke 1970

Burke 1974

Henrys $1977^{\mathrm{a}}$

\section{Parallel Breech Births}

6 breech births with SCI, one with autopsy due to death. 1 sentence states: "It was shown in the autopsy specimen that this column can be stretched 2 inches and the cervical cord pulled down 1/4 inch."

2 breech births, one of whom sustained a spinal cord injury. The authors state that x-rays of the spine were normal.

2 cases and review of 82 breech deliveries, $25 \%$ of whom had spinal cord injury associated with persistent intra-uterine neck hyper-extension at presentation. Little or no work-up was done radiologically post-partum to establish any spinal column injury.

Normal anatomy and variants (no SCI)

Entitled "the normal cervical spine in infants and children." Included is a description of the normal C2-3 pseudosubluxation phenomenon seen in adults and children at all levels when X-rayed in mid-flexion, but most pronounced at C2-3 in children.

Entitled "pseudosubluxation and other normal variations in the cervical spine in children." Authors describe C2-3 pseudo-subluxation and emphasize pitfalls and misdiagnoses of radiologic anatomy.

4 cases of children with stiff necks unable to extend due to upper respiratory infections who they state were erroneously treated with traction and bracing for their failure to recognize $\mathrm{C} 2-3$ pseudo-subluxation as a normal variant at the time.

Authors report a new classification of "dislocation" which they state is not a dislocation, but a C2-3 pseudo-subluxation normal variant, to emphasize the fact that these have been incorrectly treated. The authors $\mathrm{x}$-ray 100 normal children and show that $67 \%$ have $0-2.5 \mathrm{~mm}$ of C2-3 step-off normally and the remainder show a maximum of 3-4 mm.

Older adults with hyper-extension injury (and underlying stenosis)

Single case report of adult sustaining SCI after a hyperextension fall onto the face without radiographic spinal column injury. Pincer mechanism by which the ligamentum flavum damages the spinal cord is put forth.

45 adult patients, 37 SCIs, but 10 with normal static x-ray views, no CT or MRI. Pincer hyper-extension mechanism in older adults is reviewed.

No (or not enough ${ }^{\mathrm{a}}$ ) local static plain film evidence to explain SCI in children

7 patients, 3 with subluxations, 3 with rib fractures. Despite no MRI or CT, the author notes that no clear plain radiograph findings explain the spinal cord injury in a few. Author implicates a distraction mechanism.

25 patients (plus 5 birth injuries). Not all injuries clearly associated with a bony abnormality (no CT or MRI). Plain film myelogram done in 1 patient showed an inexplicable non-local lesion away from the neurologic level.

1,299 spine traumas, 631 cervical, 18 cervical in children < 15 years, none SCIWORA. 7 neuro-deficits, 3 tetraplegia, all with radiographic fracture-dislocation. 5 cases with radiographic lateral subluxation, 3 with neuro-deficit. Authors surmise: "it is possible, especially considering 3 of 5 patients sustained neurological injury, that some were complete dislocations with spontaneous reduction but this was impossible to determine from a retrospective study." The remaining four neurologic injuries to which the subluxation group might refer were a transient arm paresis, a decerebration, a brachial plexus palsy and a spastic cerebral palsy for which the claim does not apply.

Summary of the 11 terminal articles cited as evidence for the general and specific empirical claim (see text) made by the SCIWORA hypothesis traced to origin

apang also references Henrys et al., 1977, for the specific claim, though the authors, themselves, do not make or substantiate this claim [8, 18]

explain some SCIs (Table 1) [18-20]. The remaining citations terminated in the following 2 basic ways: (1) deadend--the terminal reference does not make or suggest the original claim being traced, (2) case series of children-some with SCI, but without a local spinal column injury seen on radiographic analysis as seen, for example, in Pang's articles [1, 8, 21-26]. A SCIWORA label is given, but no evidence for its validity. Rather, SCIWORA and differential stretch is assumed a priori without ever demonstrating its truth. Three recurrent terminal articles are illustrative and included in Table 1 [18-20].

Thus, little, if any, genuine or empirical evidence appears to exist for the SCIWORA hypothesis and its idea about the differential stretch between the spinal column and spinal cord. Rather, it starts with the assumption of locality and then infers SCIWORA when a certain radiologic modality was not sensitive enough to detect the explanation -or- nonlocality was not recognized while the explanation was present all along. In point of fact, in his original 1982 article, Pang describes a case of L2 flexion-distraction lapbelt fracture and a T6 SCIWORA [1]. This L2,T6 mismatch distraction injury was likely similar to our case 5 (L2,T9 mismatch), i.e., a non-local SCI above the spinal column uncoupling. If correct, then the L2,T6 case was neither a SCIWORA (nor a SCIWONA) since the entire column injury causing the SCI was visible on CT and MRI, just unrecognized for non-locality. There are likely many cases mislabeled throughout the literature and we wonder if difficult to accept theories about perfectly self-reducing spinal dislocation are not the product of the assumption of local effects only. Of special note, we commonly receive adults with acquired degenerative stenosis and limited space 
available for the cord who have sustained a hyperextension SCI after a simple fall. Since there is often no spinal column disruption, by definition, this may be called a SCIWORA or SCIWONA. Though true trivially and semantically, there is a rational explanation without mystery in these cases. The acquired local stenosis and sudden ligamentum flavum buckling at the extreme of physiologic motion during the fall is the culprit and has long been known [17]. Again, it is a satisfactory explanation to guide treatment that is desired. Though SCIWORA is not disputed absolutely, non-local distraction injury will often suffice once locality is relinquished. For alleged remaining SCIWORAs, an adequate explanation, not a diagnostic label, is wanting.

The clinical concern of a SCIWORA misdiagnosis is inappropriate management like over-bracing or underoperating including unnecessary cost, risk, or detriment in terms of pressure ulcer risk or encumbrance to rehabilitation of a SCI patient. Three of our six cases had SCI below the spinal column injury with risk of subsequent higher level SCI if the unstable level were not treated appropriately (three cases had complete SCI above the column injury, so neurologic risk was less critical except had they been incomplete). Misunderstanding the injuries led to overbracing and additional cost. An additional consideration is the occasional recurrence of SCIWORA reported [23, 25]. As only a peripheral theory, recurrence might signal nonlocality. If a distant spinal column injury was not treated, and did not heal, then ongoing instability may be the cause for SCI recurrence, especially in children with daily falls and play. Treatment of non-local injuries might prevent many SCIWORA recurrences.

As a point of final clarification, there are a myriad of terms which have emerged around SCIWORA, including SCIWORET (SCI without radiographic evidence of trauma), SCIWOCTET (SCI without CT evidence of trauma) and SCIWONA which are variably applied to pediatric and adult patients $[2,27,28]$. These terms appear misguided when applied to the explanation for a SCI. Rather, the studies using these terms are assessing the sensitivity of various individual radiographic modalities (e.g., static radiographs, CT, MRI, dynamic radiographs) or their combination in determining the explanation or cause for the SCI. In some cases, the explanation is a genuine spinal column injury, in others it is ligamentum flavum buckling in the setting of degenerative stenosis, or a large disc herniation, or even underlying congenital or pathologic states of the spinal column. In this way, these studies are useful to help reduce over-utilization of additional radiologic studies while understanding the limits of detection for any one modality in the evaluation of SCI patients. However, the problem is the SCI patients who seemingly have no explanation on any of our radiologic studies. To these, the SCIWORA hypothesis about the differential stretch between the spinal column and spinal cord, especially in pediatric patients, has formed and embedded itself in the literature, never having been empirically verified, but merely inferred from various case series where a certain radiologic modality was not sensitive enough to detect the explanation -or- non-locality was not recognized while the explanation was present all along.

To further complicate terminology, some authors have included the presence, absence or type of findings of cord damage on the MRI as part of the diagnostic criteria for SCIWORA [2, 8, 21]. Again, this is not relevant as what needs to be explained is not cord damage as seen on MRI, but why the clinical cord syndrome exists at all after a trauma, whether seen on MRI or not. In case 1 that we present, initial MRI did not reveal cord damage at T2 even though the patient was clinically a complete $\mathrm{T} 2$ paraplegic. Follow-up MRI at 2 weeks revealed abundant cord damage and he remained a T2 complete paraplegic. What was lacking was the explanation for the cord injury which was present as a non-local and radiologically evident spinal column disruption all along. Attempts to classify SCI recovery based on MRI cord signal findings are useful, but have no bearing on the cause of the injury in the first place or the SCIWORA hypothesis itself.

This paper has some important limitations, though we submit that once one agrees that even a single legitimate case of non-locality has occurred, then its real existence is verified. Our literature search is subject to revision through time. It may be limited by the initial 10 -year time frame selected, the keywords used, or the degree to which the authors were able to read, trace, and adequately survey, interpret, and represent the hundreds of articles examined in reference to the claims under scrutiny.

In conclusion, SCI may occur at a level noncontiguous with the causal spinal column injury occurring via a distraction mechanism, what we call non-locality. Consideration of these injuries may prevent unnecessary or incorrect bracing or treatment for presumed SCIWORA instability at the level of SCI, sparing additional cost, risk, and discomfort while fostering a proper search for (including complete spinal imaging), and treatment of, the actual causative spinal column injury which may exist elsewhere. This awareness may facilitate our search for adequate explanations for difficult to understand cases rather than mere or mysterious assignment as spinal cord injuries without (neuro)-radiographic abnormality.

\section{Compliance with ethical standards}

Conflict of interest The authors declare that they have no conflict of interest. 
Publisher's note: Springer Nature remains neutral with regard to jurisdictional claims in published maps and institutional affiliations.

\section{References}

1. Pang D, Wilberger JE Jr. Spinal cord injury without radiographic abnormalities in children. J Neurosurg. 1982;57:114-29.

2. Yucesoy K, Yuksel KZ. SCIWORA in MRI era. Clin Neurol Neurosurg. 2008;110:429-33.

3. Trigylidas T, Yuh SJ, Vassilyadi M, Matzinger MA, Mikrogianakis A. Spinal cord injuries without radiographic abnormality at two pediatric trauma centers in Ontario. Pediatr Neurosurg. 2010;46:283-9.

4. Kirshblum SC, Burns SP, Biering-Sorensen F, Donovan W, Graves DE, Jha A, et al. International standards for neurological classification of spinal cord injury (Revised 2011). J Spinal Cord Med. 2011;34:535-46.

5. Hsu AC, Norheim EP, Brambila M, Rolfe KW. T2 spinal cord injury caused by non-contiguous traumatic $\mathrm{C} 1-\mathrm{C} 2$ ligamentous injury in a young child. JBJS Case Connect. 2013;3:e25-1-4.

6. Bhalla A, Rolfe KW. T11-12 spinal column injury causing C8 tetraplegia misdiagnosed as SCIWORA in an adult. JBJS Case Connect. 2014;4:e18-1-3.

7. Fassett DR, McCall T, Brockmeyer DL. Odontoid synchondrosis fractures in children. Neurosurg Focus. 2006;20:E7.

8. Pang D. Spinal cord injury without radiographic abnormality in children, 2 decades later. Neurosurgery. 2004;55:1325-42.

9. Leventhal HR. Birth injuries of the spinal cord. J Pediatr. 1960;56:447-53.

10. Abroms IF, Bresnan MJ, Zucherman JE, Fischer EG, Strand R. Cervical cord injuries secondary to hyperextension of the head in breech presentations. Obstet Gynecol. 1973;41:369-78.

11. Bresnan MJ, Abroms IF. Neonatal spinal cord transection secondary to intrauterine hyperextension of the neck in breech presentation. J Pediatr. 1974;84:734-7.

12. Bailey DK. The normal spine in infants and children. Radiology. 1952;59:712-9.

13. Catell HS, Filtzer DL. Pseudosubluxation and other normal variants in the cervical spine in children. J Bone Jt Surg Am. 1965;47-A:1295-309.
14. Townsend EH Jr, Rowe L. Mobility of the upper cervical spine in health and disease. Pediatrics. 1952;10:567-74.

15. Sullivan CR, Bruwer AJ, Harris LE. Hypermobility of the cervical spine in children: a pitfall in the diagnosis of cervical dislocation. Am J Surg. 1958;95:636-40.

16. Marar BC. Hyperextension injuries of the cervical spine. J Bone Jt Surg Am. 1974;56-A:1655-62.

17. Taylor AR. The mechanism of injury to the spinal cord in the neck without damage to the vertebral column. J Bone Jt Surg Br. 1951;33-B:543-47.

18. Henrys P, Lyne D, Lifton C, Salciccioli G. Clinical review of cervical spine injuries in children. Clin Orthop Relat Res. 1977;129:172-6.

19. Burke DC. Spinal cord trauma in children. Paraplegia. 1971;9:1-14

20. Burke DC. Traumatic spinal paralysis in children. Paraplegia. 1974;11:268-76.

21. Grabb PA, Pang D. Magnetic resonance imaging in the evaluation of spinal cord injury without radiographic abnormality in children. Neurosurgery. 1994;35:406-14.

22. Hamilton MG, Myles ST. Pediatric spinal injury: Review of 174 hospital admissions. J Neurosurg. 1992;77:700-4.

23. Pang D, Pollack IF. Spinal cord injury without radiographic abnormality in children--the SCIWORA syndrome. J Trauma. 1989;29:654-64.

24. Osenbach RK, Menezes AH. Spinal cord injury without radiographic abnormality in children. Pediatr Neurosci. 1989;15:168-75.

25. Pollack IF, Pang D, Sclabassi R. Recurrent spinal cord injury without radiographic abnormalities in children. J Neurosurg. 1988;69:177-82.

26. Ruge JR, Sinson GP, McLone DG, Cerullo LJ. Pediatric spinal injury: The very young. J Neurosurg. 1988;68:25-30.

27. Dreizen D, Kim W, Kim JS, Boscak AR, Bodanapally UK, Munera F, et al. Will the real SCIWORA please stand up? Exploring clinicoradiologic mismatch in closed spinal cord injuries. Am J Roentenol. 2015;205:853-60.

28. Como JJ, Samia H, Nemunaitis GA, Vikas J, Anderson JS, Malangoni MA, et al. The misapplication of the term spinal cord injury without radiographic abnormality (SCIWORA) in adults. J Trauma Acute Care Surg. 2012;73:1261-6. 